\title{
Search for Kaluza-Klein gravitons in extra dimension models via forward detectors at the LHC
}

\author{
Gi-Chol Cho $^{a}$, Takanori Kono ${ }^{a}$, Kentarou Mawatari $^{b}$, Kimiko Yamashita $^{c}$ \\ ${ }^{a}$ Department of Physics, Ochanomizu University, Tokyo 112-8610, Japan \\ ${ }^{b}$ Theoretische Natuurkunde and IIHE/ELEM, Vrije Universiteit Brussel, \\ and International Solvay Institutes, Pleinlaan 2, B-1050 Brussels, Belgium \\ ${ }^{c}$ Graduate School of Humanities and Sciences, Ochanomizu University, Tokyo 112-8610, \\ Japan
}

\begin{abstract}
We investigate contributions of Kaluza-Klein (KK) graviton in extra dimension models to the process $p p \rightarrow p \gamma p \rightarrow p \gamma j X$, where a proton emits a quasireal photon and is detected by using the very forward detectors planned at the LHC. In addition to the $\gamma q$ initial state as in the Compton scattering in the standard model, the $\gamma g$ scattering contributes through the $t$-channel exchange of KK gravitons. Taking account of pileup contributions to the background and examining viable kinematical cuts, constraints on the parameter space of both the ADD (Arkani-Hamed, Dimopoulos and Dvali) model and the RS (Randall and Sundrum) model are studied. With $200 \mathrm{fb}^{-1}$ data at a centerof-mass energy of $14 \mathrm{TeV}$, the expected lower bound on the cutoff scale for the ADD model is $6.3 \mathrm{TeV}$ at $95 \%$ confidence level, while a lower limit of $2.0(0.5) \mathrm{TeV}$ is set on the mass of the first excited graviton with the coupling parameter $k / \bar{M}_{\mathrm{Pl}}=0.1(0.01)$ for the RS model.
\end{abstract}




\section{Introduction}

After the discovery of the Higgs boson [1,2], the most important task of high energy physics at the LHC is to look for signatures of new physics beyond the standard model (SM). A possibility of introducing spatial extra dimensions has been discussed to explain a large hierarchy between the Planck scale $\left(\sim 10^{18} \mathrm{GeV}\right)$ and the Fermi scale $\left(\sim 10^{2} \mathrm{GeV}\right)$. Two representative scenarios in extra dimensional models are the large extra dimension model by Arkani-Hamed, Dimopoulos and Dvali (ADD) [3,4] and the warped extra dimension model by Randall and Sundrum (RS) [5]. The main difference between the two models is the role of extra dimensions solving the hierarchy problem while a common phenomenological consequence of the two models is Kaluza-Klein (KK) excitations of graviton. Therefore searching for KK gravitons in high-energy collider experiments is crucial to probe if our spacetime is four-dimension or more.

At the LHC, signatures of ADD gravitons have been often studied in monojet [6,7] or monophoton [8,9] plus missing energy final states, where the missing energy is carried by real emissions of the KK gravitons. Moreover, ADD signatures from virtual KK graviton exchange have been sought in diphoton [10,11], dilepton [12,13] and dijet [14] final states. For the RS model, on the other hand, the resonant production of the first excitation of the KK gravitons has been looked for in diphoton [10,11], dilepton [15, 16], dijet [17] and di- $W$ boson [18, 19 final states. No significant excess over the SM background has been observed so far, setting the limit, e.g. on the Planck scale in $3+\delta$ spatial dimensions $\left(M_{D}\right)$ at around $4 \mathrm{TeV}$ with $\delta=4[\sqrt[6]{6}$ and on the scale of virtual graviton exchange at around $7 \mathrm{TeV} 14$ for the ADD model, and on the first KK graviton mass at around $2.7 \mathrm{TeV}$ with the coupling parameter $k / \bar{M}_{\mathrm{Pl}}=0.1$ for the RS model [16]. Thus an expectation to find the signal of KK graviton is postponed until the next (or future) stage of LHC experiments such as $p p$ collision in $\sqrt{s}=13 \mathrm{TeV}$ or at the high-luminosity LHC with an integrated luminosity $L_{\text {int }}=3000 \mathrm{fb}^{-1}$. Among the upgrade plans of LHC experiments, the installation of very forward detectors in addition to the central detectors to study the $p p$ scattering with very large pseudorapidity of protons is proposed by ATLAS and CMS [20]. The former is called ATLAS Forward Physics (AFP) project, and the latter is called CMS-TOTEM forward detector scenario (CMS-TOTEM, in short). The forward detectors will be set at $220 \mathrm{~m}$ and $420 \mathrm{~m}$ from the interaction point in the AFP project while it is set at $420 \mathrm{~m}$ from the interaction point in addition to the TOTEM detectors in the CMS-TOTEM scenario. These forward detectors will detect the scattered protons which have momentum fraction loss

$$
\xi \equiv \frac{|\boldsymbol{p}|-\left|\boldsymbol{p}^{\prime}\right|}{|\boldsymbol{p}|}
$$

where $\boldsymbol{p}$ and $\boldsymbol{p}^{\prime}$ denote the three momenta of incoming and outgoing protons, respectively. The acceptance of $\xi$ is aimed as $0.0015<\xi<0.15$ for AFP and $0.0015<\xi<0.5$ for 


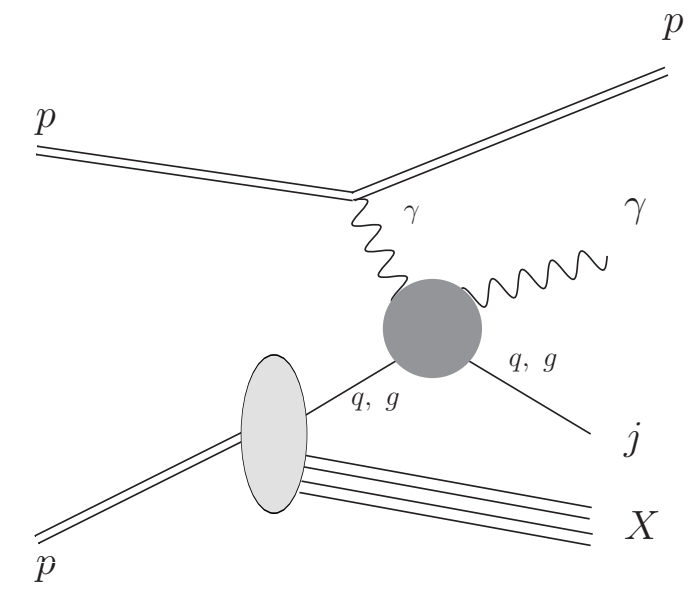

Figure 1: Schematic diagram for $p p \rightarrow p \gamma p \rightarrow p \gamma j X$.

\section{CMS-TOTEM.}

In this paper, we study the possibility of using the forward detectors to find indirect constraints on the KK graviton in both the ADD and RS models through the $t$-channel KK graviton exchange effects in

$$
p p \rightarrow p \gamma p \rightarrow p \gamma j X
$$

The schematic diagram is shown in Fig. 1. In this process, a quasireal photon $\gamma$ with low virtuality is emitted from a proton which scatters with a parton in the proton coming from the opposite direction. The proton which emits $\gamma$ is scattered with very small angle without dissociation into partons, and detected by the forward detectors. An earlier work to study the KK graviton in the above process has been presented in Ref. [21], where the authors obtained the bounds on KK graviton from $\gamma q \rightarrow \gamma q$ ( $q$ is a quark or antiquark in a proton) processes performing a simple parton-level analysis. It must be noted that the process $\gamma q \rightarrow \gamma q$ is the only subprocess of the process (2) at the leading order in the SM. However, the exchange of the KK gravitons allows $\gamma g \rightarrow \gamma g$ as another subprocess. In our study, therefore, we revisit the KK graviton contributions to the process (2) including all possible subprocesses. Moreover, we take into account the SM background coming from pileup events, whose contribution can be significant in the future higher-energy run, pointed out recently in Ref. [22]. We study constraints on the parameter space of ADD and RS models by examining viable kinematical cuts and taking account of parton-shower and hadronization effects in the final states as well as detector effects to find more realistic bounds on the models, which have not been discussed in Ref. [21].

This paper is organized as follows. In Sec. 2, we give a brief review of the ADD and RS models to fix our notation. The results of numerical analysis are given in Sec. 3 . Section 4 is devoted to summary of our findings. 


\section{Models}

In this section we briefly review the ADD [3] and RS [5] models, focusing on interactions of KK gravitons to the SM fields.

In the both extra dimension models, there appear KK towers of gravitons, and the effective interaction Lagrangian with the SM fields is given by 23, 24

$$
\mathcal{L}=-\frac{1}{\Lambda} T^{\mu \nu}(x) \sum_{n} h_{\mu \nu}^{(n)}(x),
$$

where $T^{\mu \nu}$ is the energy-momentum tensor of the SM fields (see, e.g., Ref. 25] for the explicit forms), $h_{\mu \nu}^{(n)}$ is the $n$th KK mode of the graviton, and $\Lambda$ is the relevant interaction scale.

The ADD model is given in the $(4+\delta)$-dimensional spacetime where the extra $\delta$-dimensions are compactified on a torus $T^{\delta}$ with a common radius $R$. The four-dimensional Planck scale $M_{\mathrm{Pl}}$ is related to the $(4+\delta)$-dimensional fundamental scale $M_{D}$ as $M_{\mathrm{Pl}}^{2} \sim M_{D}^{2+\delta} R^{\delta}$ owing to the Gauss's law. All the SM fields are expected to be confined on the four-dimensional spacetime and only the gravitational interaction propagates into the extra dimensions. The scale of the interaction in Eq. (3) is given by

$$
\Lambda=\bar{M}_{\mathrm{Pl}} \equiv M_{\mathrm{Pl}} / \sqrt{8 \pi} \approx 2.4 \times 10^{18} \mathrm{GeV}
$$

where $\bar{M}_{\mathrm{Pl}}$ is the reduced four-dimensional Planck scale. Setting the fundamental scale $M_{D}$ to be $1 \mathrm{TeV}$, two extra dimensions $\delta=2$ imply a large size of radius $R \sim \mathcal{O}(0.1 \mathrm{~mm})$. After compactification of extra dimensions, there are KK excitations of gravitons whose spacing is given by $\sim 1 / R$. This leads to infinity in the KK graviton propagator after summing up all KK modes and we replace the propagator by the cut-off parameter $\Lambda_{T}$ (of order $M_{D}$ ) for $\delta>2$ by 23

$$
\frac{1}{\bar{M}_{\mathrm{Pl}}^{2}} \sum_{n} \frac{1}{q^{2}-m_{n}^{2}} \equiv \frac{4 \pi}{\Lambda_{T}^{4}},
$$

where $m_{n}$ is the mass of the $n$th KK mode of the graviton ${ }^{1}$

The RS model is a five-dimensional model where one warped spatial dimension $y$ is compactified on $S^{1} / Z_{2}$ orbifold. The metric is given by

$$
d s^{2}=e^{-2 k|y|} \eta_{\mu \nu} d x^{\mu} d x^{\nu}-d y^{2}
$$

where $\eta_{\mu \nu}(\mu, \nu=0,1,2,3)$ and $k$ denote the Minkowski metric and the $\mathrm{AdS}_{5}$ curvature, respectively. The model has two $D_{3}$ branes at $y=0$ and $\pi r_{c}$, the former is called the Planck brane and the latter is called the TeV brane. The SM fields are confined in the TeV

\footnotetext{
${ }^{1}$ This cutoff scheme is the so-called Giudice-Rattazzi-Wells convention 23, while there are other conventions, e.g. by Han-Lykken-Zhang [24] and Hewett [26]. See Ref. 13] for more details.
} 
brane and only graviton propagates into the fifth dimension $2^{2}$ With this setup, the hierarchy between the Planck $\left(M_{\mathrm{Pl}}\right)$ and the Fermi $\left(M_{W}\right)$ scales is explained when $k r_{c} \simeq 12$, and the scale of the interaction in Eq. (3) is

$$
\Lambda=\Lambda_{\pi} \equiv e^{-k \pi r_{c}} \bar{M}_{\mathrm{Pl}} .
$$

Therefore the interactions of all the KK gravitons to the SM fields are suppressed by $\Lambda_{\pi} \sim$ $\mathcal{O}(\mathrm{TeV})$. The mass of the $n$th $\mathrm{KK}$ mode of the graviton is given by 27

$$
m_{n}=k x_{n} e^{-k \pi r_{c}},
$$

where $x_{n}$ is a root of the Bessel functions of the first kind. The denominator of the graviton propagator is normal:

$$
\sum_{n} \frac{1}{q^{2}-m_{n}^{2}+i m_{n} \Gamma_{n}},
$$

where $\Gamma_{n}$ denotes the graviton decay width 24.3 For the parameter scan, we use

$$
\beta=k / \bar{M}_{\mathrm{Pl}} \quad \text { and } \quad m_{G}=m_{1},
$$

which are commonly chosen.

\section{Numerical Analysis}

\subsection{Signals}

Based on the RS graviton implementation [29], we implemented the propagator in Eq. (5) for the ADD gravitons by modifying the UFO file [30 32], while we introduced higher KK graviton modes for the RS model into FeynRules [33]. We use MAdGraPh5_AMC@NLO [34] to generate parton-level events both for the signal and the SM background by employing the MSTW2008 PDF [35] through the LHAPDF interface [36], with the factorization scale fixed at five times the $Z$-boson mass $4^{4}$ We use the flux of quasireal photons emitted from a proton in MADGRAPH5_AMC@NLO, implemented in a similar manner with PDFs [37, and detail it in the appendix. The partonic events are passed to PYTHIA8 [38] for parton shower and hadronization.

We conduct analyses for the LHC at $\sqrt{s}=14 \mathrm{TeV}$. As minimal event selections, we impose cuts on the transverse momentum $p_{T}$ and the pseudorapidity $\eta$ for the photon and the leading jet as

$$
p_{T}^{\gamma}>40 \mathrm{GeV}, \quad\left|\eta^{\gamma}\right|<2.5, \quad p_{T}^{j}>50 \mathrm{GeV}, \quad\left|\eta^{j}\right|<3.0,
$$

\footnotetext{
${ }^{2}$ There have been some variants of the RS model in which some of the SM fields are allowed to propagate into the bulk. But we do not discuss such possibilities further in this paper.

${ }^{3}$ The widths are computed by the decay package 28] for each parameter point. The values are, e.g. $\Gamma_{1,2,3,4}=4.2,26,80,180 \mathrm{GeV}$ for $\left(\beta, m_{G}\right)=(0.05,1.2 \mathrm{TeV})$.

${ }^{4}$ See Ref. 21] for a more detailed discussion on the scale choices.
} 

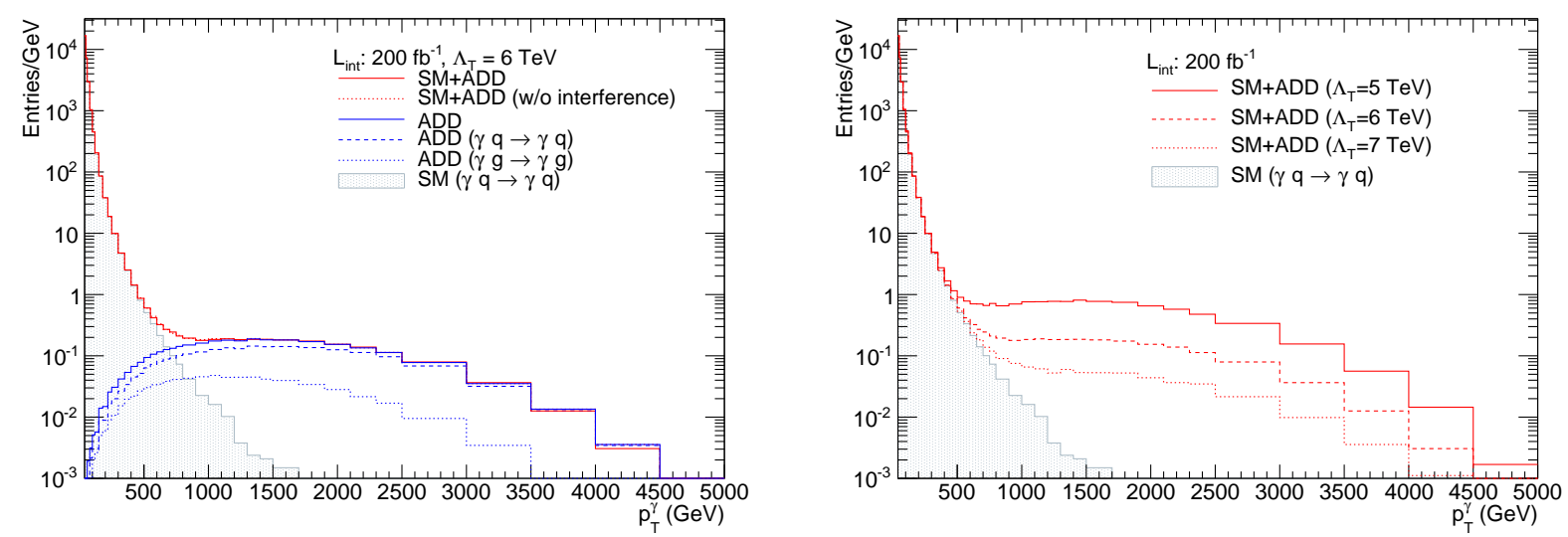

Figure 2: Left: Photon transverse momentum distribution of the signal plus background for the ADD model with the cutoff scale $\Lambda_{T}=6 \mathrm{TeV}$ in $p p \rightarrow p \gamma p \rightarrow p \gamma j X$ at $\sqrt{s}=14 \mathrm{TeV}$. The contributions of each subprocess are also shown. Right: The $p_{T}^{\gamma}$ distributions for $\Lambda_{T}=5$, 6 and $7 \mathrm{TeV}$.

where jets are reconstructed by the anti- $k_{T}$ algorithm [39] with a distance parameter of 0.6. The jet-energy resolution is set to $10 \%$. To show our results, we assume the integrated luminosity $L_{\text {int }}=200 \mathrm{fb}^{-1}$, and take into account the so-called survival probability $S=0.7$ that the proton remains intact and the detection efficiency of the photon of $\varepsilon_{\gamma}=0.8$. Therefore, the number of events is $N=\sigma \times L_{\text {int }} \times S \times \varepsilon_{\gamma}$.

In Fig. 2 we show the $p_{T}$ distribution of the photon for the ADD model with the cutoff scale $\Lambda_{T}=6 \mathrm{TeV}$. Here, we generate events for the signal (blue lines) and the background (shaded) independently, and compare with the full sample (red solid) including the interference between them. While only the $\gamma q$ initial state contributes in SM, the $\gamma g$ scattering contributes in extra dimension models, leading to about $25 \%$ enhancement of the ADD signal rate. The signal dominates in the high- $p_{T}$ region, and therefore a certain $p_{T}^{\gamma}$ cut largely reduces the background. We note that the interference between the signal and background is very small. In Fig. 2(right) we show the $p_{T}^{\gamma}$ distributions of the signal plus background for $\Lambda_{T}=5,6$ and $7 \mathrm{TeV}$.

Similar to the ADD case, the high- $p_{T}$ photons are expected in the RS case, shown in Fig. 3(left), where a benchmark $\left(\beta, m_{G}\right)=(0.05,1.2 \mathrm{TeV})$ is taken. In this case, the $n=1-4$ KK graviton masses are 1.2, 2.2, 3.2 and $4.2 \mathrm{TeV}$, respectively, and the cutoff scale $\Lambda_{\pi}$ is $6.3 \mathrm{TeV}$. The contribution from the $\gamma g$ initial state enhances the signal by about $25 \%$. In Fig. 3(right) we also show the $p_{T}^{\gamma}$ distributions of the signal plus background for $m_{G}=0.9$, 1.2 and $1.5 \mathrm{TeV}$.

In the following analyses, we impose the high- $p_{T}$ photon selection cut

$$
p_{T}^{\gamma}>600 \mathrm{GeV}
$$

to remove the background. 

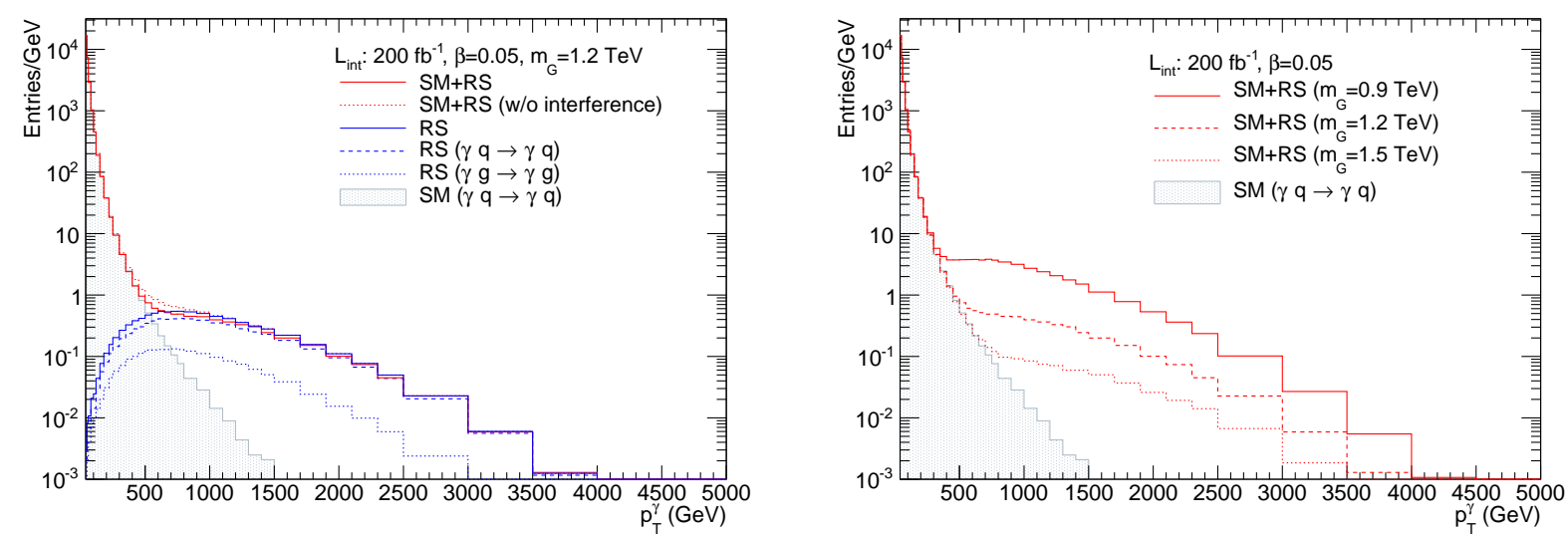

Figure 3: Same as Fig. 2, but for the RS model with $\beta=0.05$ and $m_{G}=1.2 \mathrm{TeV}$ (left) and $m_{G}=(0.9,1.2,1.5) \mathrm{TeV}$ (right).

\subsection{Background from pileup events}

At the naive parton level, the photon-induced Compton $\gamma q \rightarrow \gamma q$ process only contributes to the background. However, the $q g \rightarrow \gamma q$ and $q \bar{q} \rightarrow \gamma g$ processes, denoted as $p p \rightarrow \gamma j X$, can contribute rather significantly to the background as seen below. The LHC experiments operate under a very high-luminosity condition such that the multiple proton-proton interactions (pileup) take place in the same bunch crossing. The average number of interactions per bunch crossing $(\mu)$ around 50 is expected in the Run-II operation [40]. The majority of the pileup events consists of elastic scattering, single and double diffractive scattering and inelastic scattering. Although the forward proton is absent in $p p \rightarrow \gamma j X$, the pileup events may produce forward protons in the final state which overlap with the hard scattering events. Therefore, the following two SM processes are considered as the background in this study:

1. photon induced process $\gamma p \rightarrow \gamma j X$ (referred to $\gamma p \rightarrow \gamma+j$ ),

2. overlap between $p p \rightarrow \gamma j X$ and pileup events (referred to $p p \rightarrow \gamma+j+\mathrm{PU}$ ).

In order to evaluate the effect of pileup events, minimum bias events were generated with Pythia8 [38]. The average number of pileup events is assumed to be 50, and the overlap of $p p \rightarrow \gamma j X$ and pileup events are simulated by randomly taking multiple pileup events from the minimum bias sample for each $p p \rightarrow \gamma j X$ event. The cross section of the second process is about $16 \mathrm{nb}$ at $\sqrt{s}=14 \mathrm{TeV}$, four orders of magnitude larger than the first one (about $3 \mathrm{pb}$ ). This is expected for the hard interactions involved in these processes as the gluon-initiated process is dominant compared to photon-initiated process at the LHC. With 50 pileup events on average, there are always multiple protons in the forward region either from diffractive production or inside the proton remnant in case of inelastic scattering.

One way to reduce the $\gamma+j+\mathrm{PU}$ contribution is to use the fact that the forward proton and the particles in the main detector (photon and jet) are produced by different proton- 


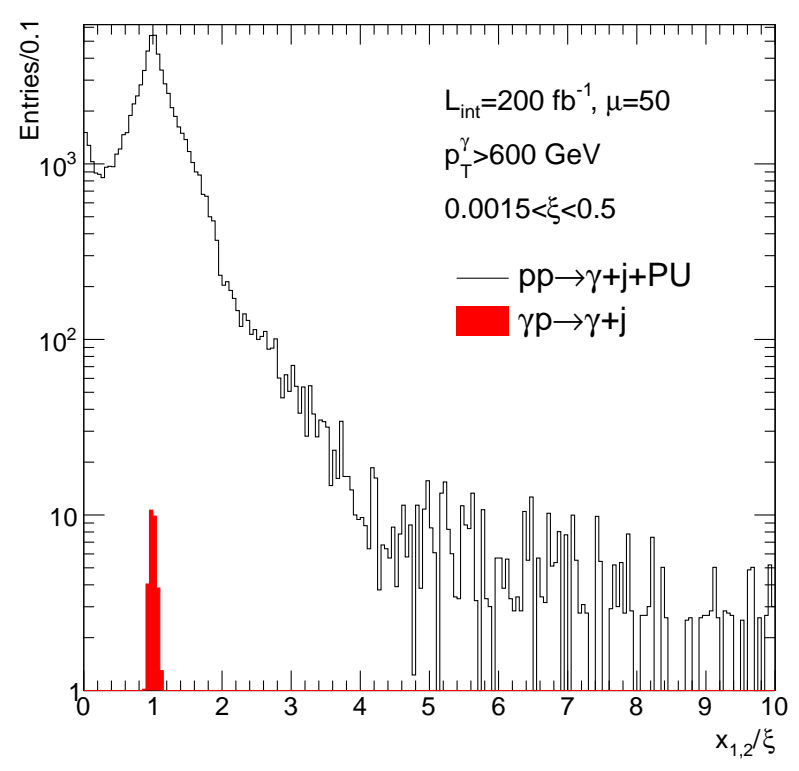

Figure 4: Distributions of $x_{1,2} / \xi$ for the overlapping events of $p p \rightarrow \gamma j X$ and pileup contribution (solid line), and the $\gamma p \rightarrow \gamma j$ sample (filled histogram), where $p_{T}^{\gamma}>600 \mathrm{GeV}$ and $0.0015<\xi<0.5$ are imposed.

proton interactions. In order to investigate this correlation, the momentum fraction of the proton taken by the partons $\left(x_{1}\right.$ and $\left.x_{2}\right)$ is calculated from the four momenta of the photon and the jet reconstructed in the main detector as

$$
x_{1}=\frac{1}{2 E_{p}}\left(p_{T}^{\gamma} e^{\eta^{\gamma}}+p_{T}^{j} e^{\eta^{j}}\right), \quad x_{2}=\frac{1}{2 E_{p}}\left(p_{T}^{\gamma} e^{-\eta^{\gamma}}+p_{T}^{j} e^{-\eta^{j}}\right) .
$$

Here, $E_{p}$ is the energy of the proton beam. The variables $x_{1}$ and $x_{2}$ correspond to the momentum fractions for the partons moving toward positive and negative $z$ direction, respectively.

For the $\gamma+q \rightarrow \gamma+q$ process, since the photon, jet and the forward proton originate from the same interaction, one expects either $x_{1} / \xi \simeq 1$ or $x_{2} / \xi \simeq 1$, where $\xi$ is defined in Eq. (1). On the other hand, if they come from different interactions, $x_{1}$ and $x_{2}$ have no correlation with $\xi$. Figure 4 shows the distribution of the ratio $x_{1,2} / \xi$ for the two background processes after requiring the minimal selection cuts (11) and the $p_{T}^{\gamma}>600 \mathrm{GeV}$ cut (12). The forward protons are required to have $0.0015<\xi<0.5$. While there is a good correlation for the $\gamma p \rightarrow \gamma+j$ process which creates a narrow peak at one, the $p p \rightarrow \gamma+j+\mathrm{PU}$ sample shows a broader spectrum. Although there should not be a correlation between $x_{1,2}$ and $\xi$ in the $p p \rightarrow \gamma+j+\mathrm{PU}$ sample, the distribution shows a peak around one. This is because the distribution is produced by taking the proton which gives the $x_{1} / \xi$ or $x_{2} / \xi$ value closest to unity if multiple protons are present in the forward region, and indeed there are several protons within this $\xi$ range with $\mu=50$. We require the event to have a forward proton 

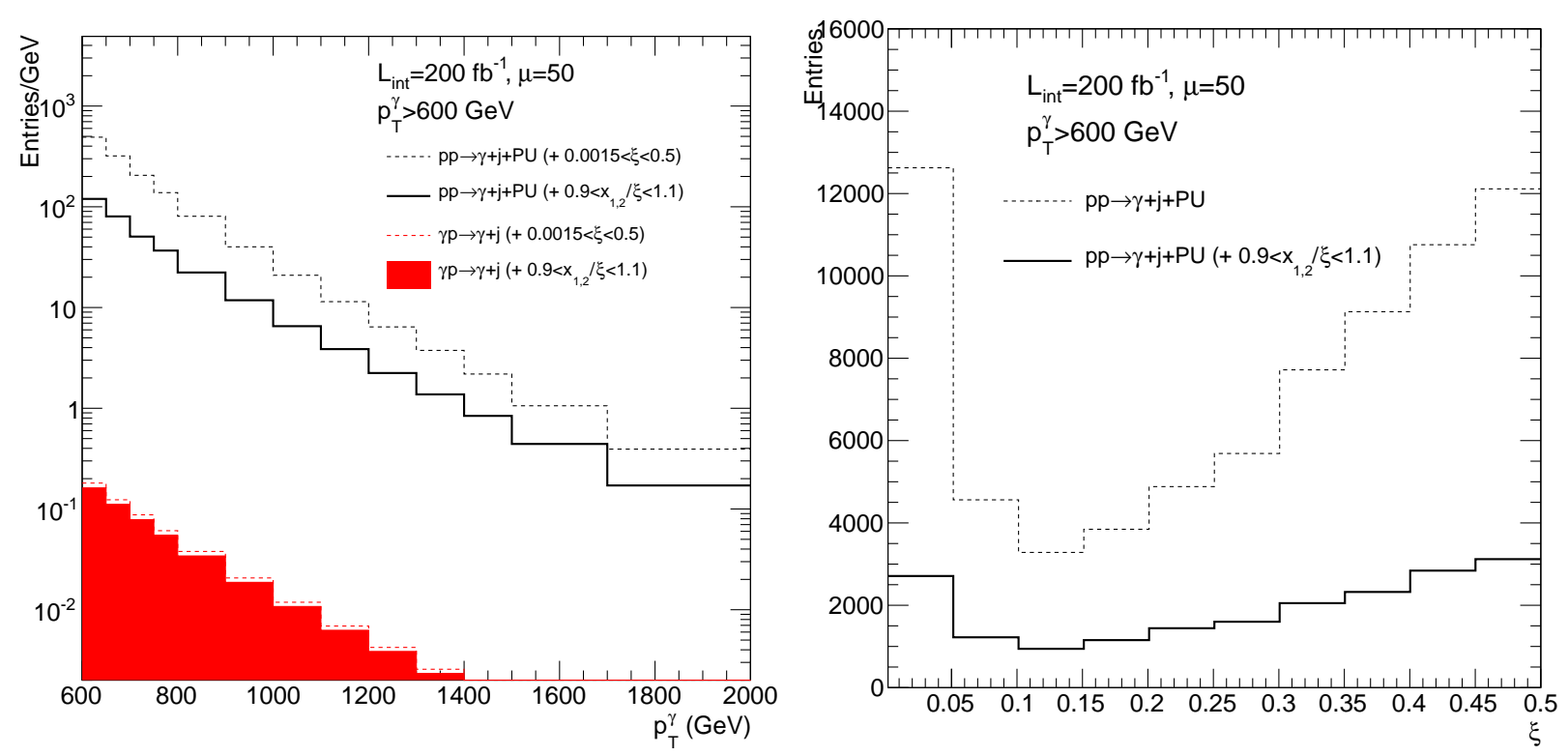

Figure 5: The $p_{T}^{\gamma}$ (left) and $\xi$ (right) distributions for the $\gamma+j+\mathrm{PU}$ and $\gamma+j$ samples with the $0.0015<\xi<0.5$ cut plus the $x_{1,2} / \xi$ ratio cut, where $p_{T}^{\gamma}>600 \mathrm{GeV}$ is imposed. For the $\xi$ distribution, only the $\gamma+j+\mathrm{PU}$ sample is shown.

\begin{tabular}{l|rrr}
\hline$\xi$ range & $\xi$ range cut & $x_{1,2} / \xi$ ratio cut & overall \\
\hline$(0.0015,0.5)$ & 1.0 & 3.9 & 3.9 \\
$(0.0015,0.15)$ & 1.2 & 13 & 16 \\
$(0.1,0.5)$ & 1.0 & 4.5 & 4.5 \\
$(0.1,0.15)$ & 4.2 & 12 & 48 \\
\hline
\end{tabular}

Table 1: Rejection factors for the $\gamma+j+\mathrm{PU}$ by applying the $\xi$ range cut and $0.9<x_{1,2} / \xi<$ 1.1. The factors are calculated with respect to the $\gamma+j+\mathrm{PU}$ sample with $p_{T}^{\gamma}>600 \mathrm{GeV}$.

with either

$$
0.9<x_{1} / \xi<1.1 \quad \text { or } \quad 0.9<x_{2} / \xi<1.1
$$

Figure 5 illustrates the effect of the $x_{1,2} / \xi$ ratio cut in Eq. (14) in the $p_{T}^{\gamma}$ (left) and $\xi$ (right) distributions, where the selection of the photon $p_{T}^{\gamma}>600 \mathrm{GeV}$ is applied. Figure 5(right) represents the $\xi$ distribution of the protons in pileup events, which are produced by diffractive scattering (small $\xi$ value) or by the hadronization into a proton from particles in the proton remnant in case of inelastic scattering (large $\xi$ value). In Fig. 5 we take the $\xi$ range cut as $0.0015<\xi<0.5$ as a representative case, while we show the rejection factors for the $\gamma+j+\mathrm{PU}$ sample with the different $\xi$-range cuts in Table 1 . With this $x_{1,2} / \xi$ selection, the $\gamma+j+\mathrm{PU}$ background is reduced by a factor of $4-50$, depending on the $\xi$ range cut, while the photon-induced $\gamma+j$ background mostly remains. 

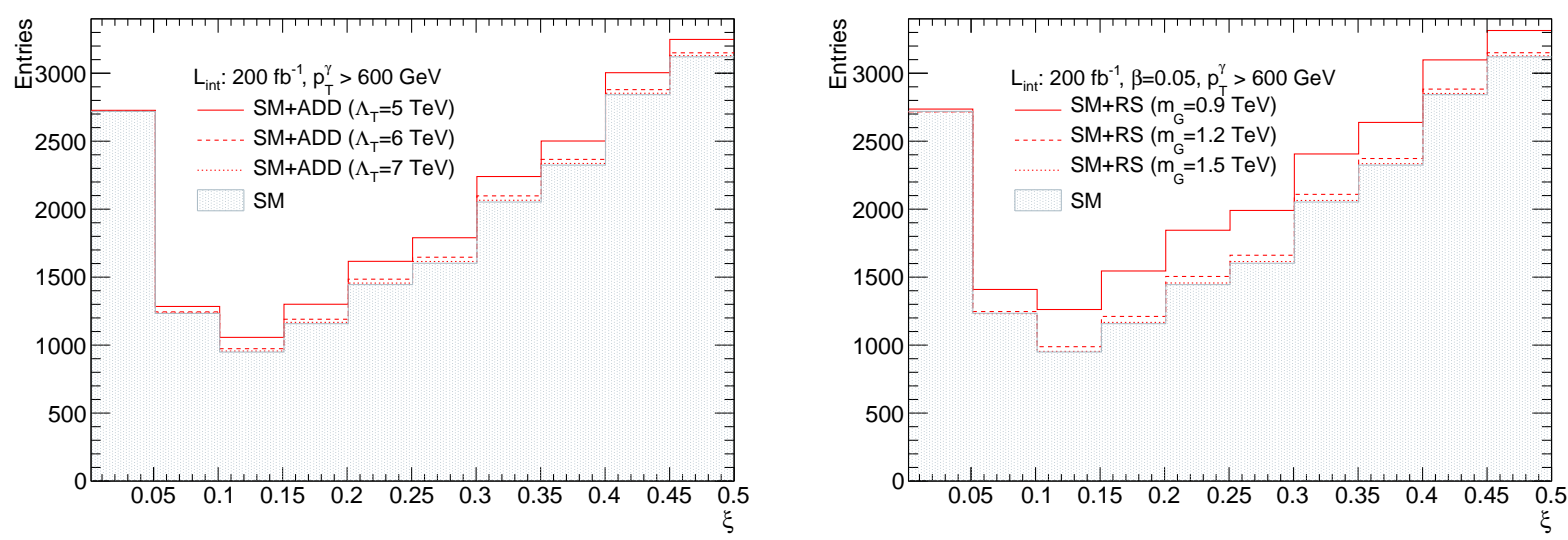

Figure 6: The $\xi$ distributions of the signal plus background for the ADD (left) and RS (right) models in $p p \rightarrow p \gamma j X$ at $\sqrt{s}=14 \mathrm{TeV}$, including the pileup contribution to the background, where $p_{T}^{\gamma}>600 \mathrm{GeV}$ and $0.9<x_{1,2} / \xi<1.1$ are imposed.

\subsection{Kinematical distributions}

Including the pileup contribution as the SM background, in Fig. 6 we show the $\xi$ distributions for the signal plus background after imposing the high- $p_{T}$ photon selection cut 12 and the $x_{1,2} / \xi$ ratio cut (14). For some lower $\Lambda_{T}$ in the ADD model and smaller $m_{G}$ in the RS model, the signal rates now become visible over the SM background. Since the graviton signals distribute mostly in the $\xi>0.1$ region, in the following analyses we impose the lower $\xi$ cut for the further background reduction as

$$
\begin{array}{ll}
0.1<\xi<0.5 & \text { for the CMS-TOTEM, } \\
0.1<\xi<0.15 & \text { for the AFP. }
\end{array}
$$

The $p_{T}$ distributions of the photon after all the event selections for the ADD and RS models are shown in Figs. 7 and 8, respectively, where we take the above different $\xi$ ranges. These illustrate that the SM background contribution is suppressed to a reasonable level. We note that, although we fixed the $p_{T}^{\gamma}$ selection cut at $600 \mathrm{GeV}$ in this work, some optimizations may help to reduce the background further.

\subsection{Limits on the model parameters}

Finally, we would like to constrain the parameter space for each model. The expected exclusion limit on the parameter space in each model is derived by assuming a null observation. Taking into account only the statistical uncertainty, the $\chi^{2}$ function is defined from the number of signal and SM background events as $\chi^{2}=\left(N_{S+B}-N_{B}\right)^{2} / N_{B}$. The number of events are normalized to integrated luminosities from $20 \mathrm{fb}^{-1}$ to $200 \mathrm{fb}^{-1}$ for the ADD model, which has only one model parameter, i.e. the cutoff scale $\Lambda_{T}$. For the RS model, on the other hand, 

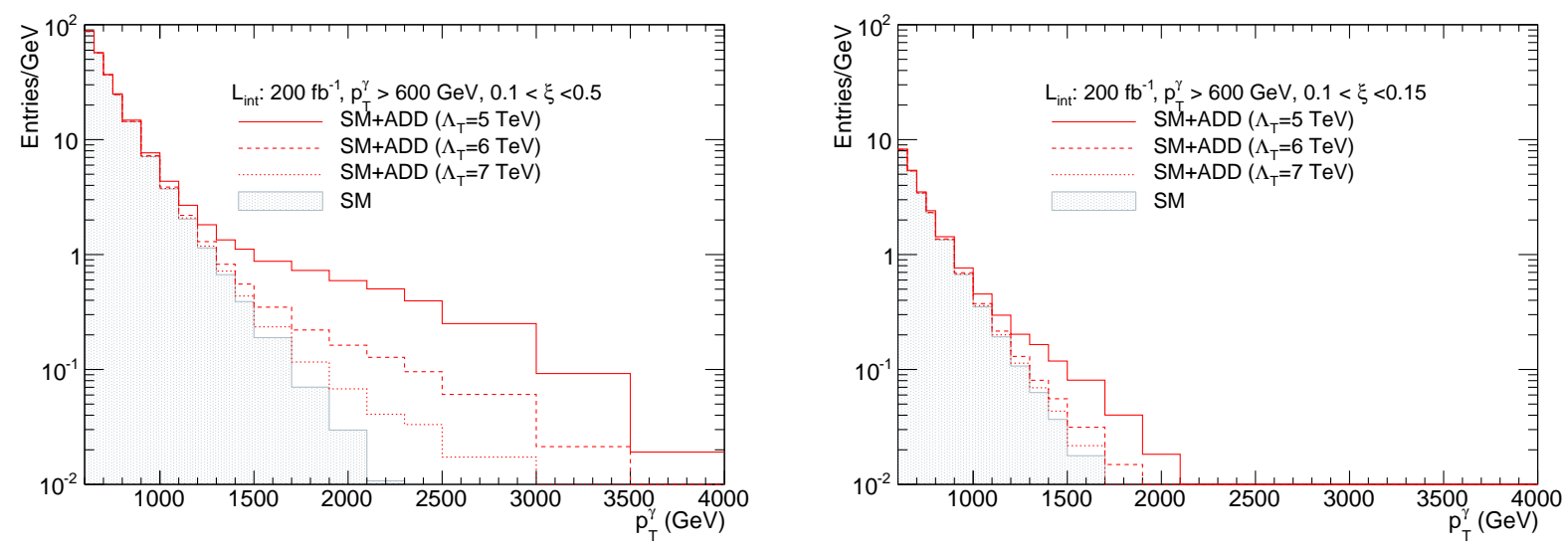

Figure 7: The $p_{T}^{\gamma}$ distributions of the signal plus background for the ADD model with the different cutoff scales, $\Lambda_{T}=5,6$ and $7 \mathrm{TeV}$, in $p p \rightarrow p \gamma j X$ at $\sqrt{s}=14 \mathrm{TeV}$ for the CMS-TOTEM (left) and the AFP (right), where $p_{T}^{\gamma}>600 \mathrm{GeV}$ and $0.9<x_{1,2} / \xi<1.1$ are imposed.
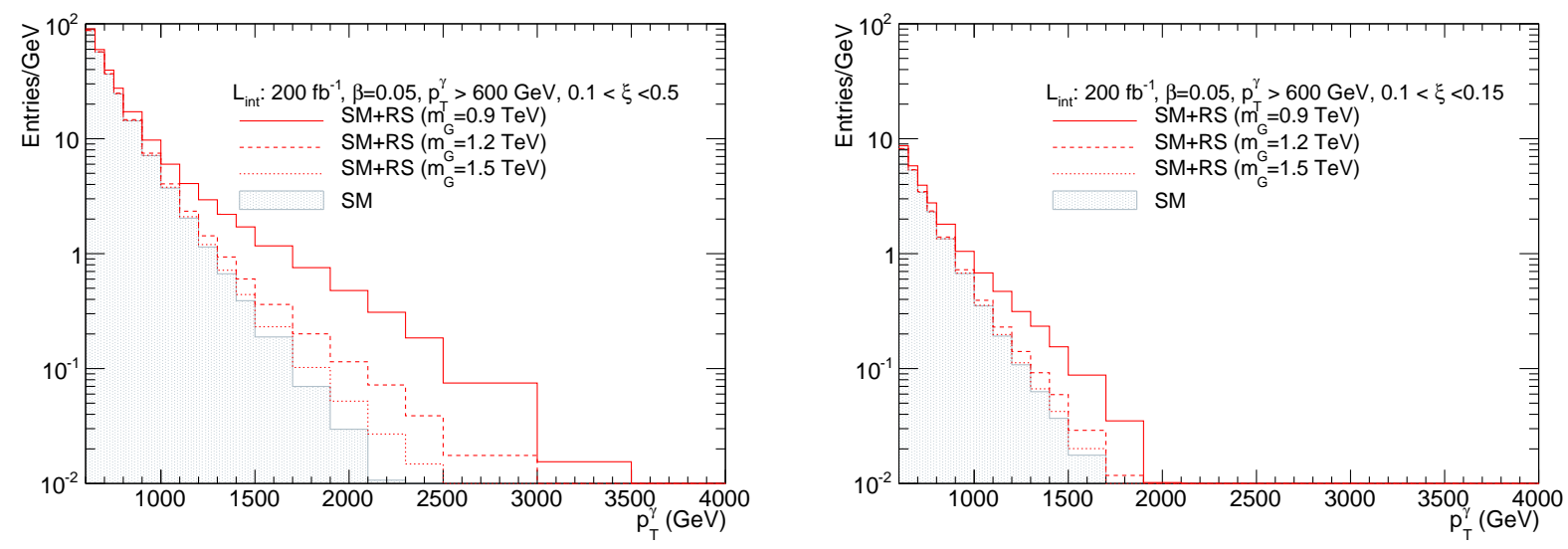

Figure 8: Same as Fig. 7, but for the RS model with the fixed coupling parameter $\beta=0.05$ and the different KK graviton masses, $m_{G}=0.9,1.2$ and $1.5 \mathrm{TeV}$.

we fix an integrated luminosity at $200 \mathrm{fb}^{-1}$ and scan the two model parameters, i.e. $\beta$ and $m_{G}$ in Eq. 10 . The model parameters are considered as excluded at $95 \%$ confidence level (CL) if $\chi^{2}>3.84$ is satisfied.

The $\chi^{2}$ is calculated for each point in the parameter space where the signal event was generated. The exclusion limit is determined at the parameter value crossing $\chi^{2}=3.84$, assuming that the $\chi^{2}$ varies smoothly with the model parameters. The uncertainty of expected exclusion limit is evaluated by varying $N_{S+B}$ and $N_{B}$ within their Poisson uncertainties, and repeating the procedure to obtain the limit. With the kinematic selection described above, $N_{B}=13246$ (1245) for $0.1<\xi<0.5(0.1<\xi<0.15)$ is obtained for an integrated luminosity of $200 \mathrm{fb}^{-1}$ which allows a reasonable estimate of the statistical uncertainty.

The expected lower bound of $\Lambda_{T}$ for the ADD model is shown in Fig. 9 as a function 


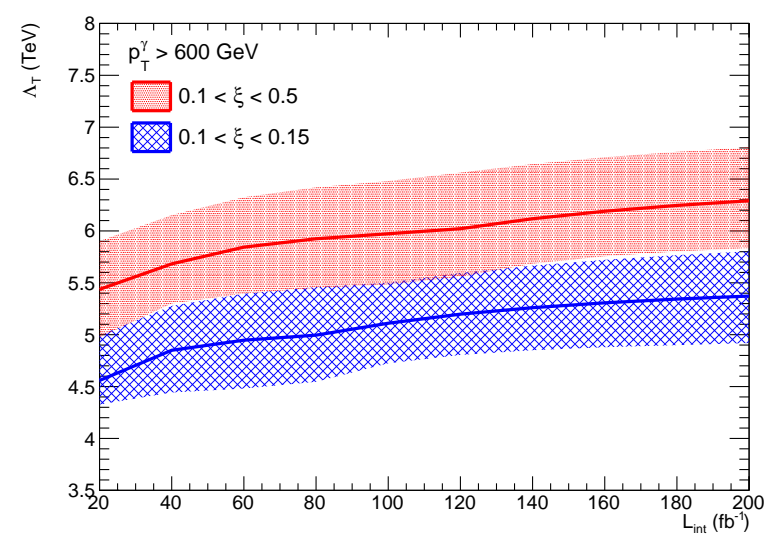

Figure 9: The $95 \%$ CL lower bound on the cutoff parameter $\Lambda_{T}$ in the ADD model for $0.1<\xi<0.5$ (red, CMS-TOTEM) and $0.1<\xi<0.15$ (blue, AFP) as a function of the integrated luminosity. The shaded bands around the limit indicate the $\pm 1 \sigma$ uncertainties.

of an integrated luminosity. With $200 \mathrm{fb}^{-1}$ data, a lower bound of $\Lambda=6.3$ (5.4) $\mathrm{TeV}$ can be achieved for the CMS-TOTEM (AFP). Selecting events in $0.1<\xi<0.5$ gives a better results compared to a narrower range $0.1<\xi<0.15$. These limits are comparable with the current dijet analysis [14], where the $s$-channel virtual gravitons are considered.

The exclusion limit for the RS model is shown in Fig. 10, where an integrated luminosity of $200 \mathrm{fb}^{-1}$ is assumed. Again, a more stringent limit is obtained by using the $\xi$ range cut of $0.1<\xi<0.5$ (CMS-TOTEM). A 95\% CL lower limit on the first KK graviton mass for the CMS-TOTEM is expected to be $2.0(0.5) \mathrm{TeV}$ for the coupling parameter $\beta=0.1$ (0.01), which is slightly weaker than the current limit from the dilepton analysis [16]. The limit for the AFP is weaker and the values are $1.7(0.4) \mathrm{TeV}$ for $\beta=0.1(0.01)$.

\section{Summary}

We have studied possibilities to search for the KK graviton in the process $p p \rightarrow p \gamma p \rightarrow$ $p \gamma j X$ using the very forward detectors planned at the LHC. This process consists of two subprocesses $\gamma q \rightarrow \gamma q$ and $\gamma g \rightarrow \gamma g$, where the latter appears only through the $t$-channel KK graviton exchange and has been overlooked in the previous study [21]. We examined all possible subprocesses and viable kinematical cuts to reduce the SM background to the signal events.

A serious background to the signal process besides the SM Compton process comes from the overlap between hard scatterings, $q g \rightarrow \gamma q$ and $q \bar{q} \rightarrow \gamma g$, and forward protons from the pileup events. We showed that such background events could be reduced by requiring a ratio of $x_{1} / \xi$ (or $x_{2} / \xi$ ) to be between 0.9 and 1.1 .

Taking account of parton-shower and hadronization effects in the final state, we found 


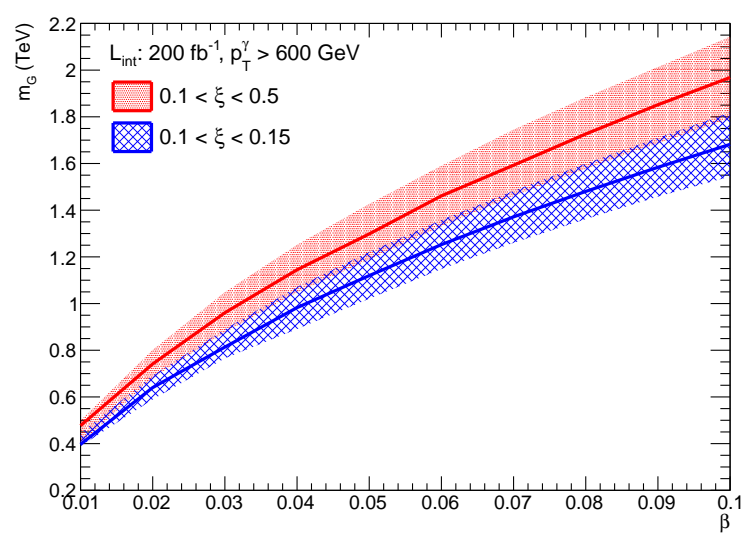

Figure 10: The $95 \%$ CL lower bound on the first KK graviton mass $m_{G}$ as a function of the coupling parameter $\beta=k / \bar{M}_{P l}$ in the RS model for an integrated luminosity of $200 \mathrm{fb}^{-1}$. The results are shown for $0.1<\xi<0.5$ (red, CMS-TOTEM) and $0.1<\xi<0.15$ (blue, AFP). The shaded bands around the limit indicate the $\pm 1 \sigma$ uncertainties.

realistic constraints on the model parameter space of ADD and RS models for the $14-\mathrm{TeV}$ LHC. The $95 \%$ CL lower bound on the cutoff scale $\Lambda_{T}$ in the ADD model is expected to be $6.3 \mathrm{TeV}$ with the $200 \mathrm{fb}^{-1}$ data for the CMS-TOTEM (5.4 TeV for the AFP). For the RS model, the lower bound of the first KK-graviton mass $m_{G}$ is $2.0 \mathrm{TeV}(0.5 \mathrm{TeV})$ with $k / \bar{M}_{\mathrm{Pl}}=0.1(0.01)$ for the CMS-TOTEM. Those for the AFP is $1.7 \mathrm{TeV}(0.4 \mathrm{TeV})$ with $k / \bar{M}_{\mathrm{Pl}}=0.1(0.01)$.

The process we studied in this article is not the conventional $s$-channel KK graviton productions, and hence the very forward detectors at the LHC might give us a new and complementary opportunity to search for new physics beyond the SM.

\section{Acknowledgements}

K. M. would like to thank the KEK theory group and Ochanomizu University for the warm hospitality, where a final part of this work was done. K. M. is supported in part by the Belgian Federal Science Policy Office through the Interuniversity Attraction Pole P7/37, and by the Strategic Research Program "High Energy Physics" and the Research Council of the Vrije Universiteit Brussel.

\section{Appendix: Photon flux from a proton}

The quasireal photon flux from a proton is described by the equivalent photon approximation (EPA) as 41]

$$
f\left(\xi, Q_{\max }^{2}\right)=\frac{\alpha_{\mathrm{EM}}}{\pi} \frac{(1-\xi)}{\xi}\left[\varphi\left(\xi, \frac{Q_{\max }^{2}}{Q_{0}^{2}}\right)-\varphi\left(\xi, \frac{Q_{\min }^{2}}{Q_{0}^{2}}\right)\right]
$$



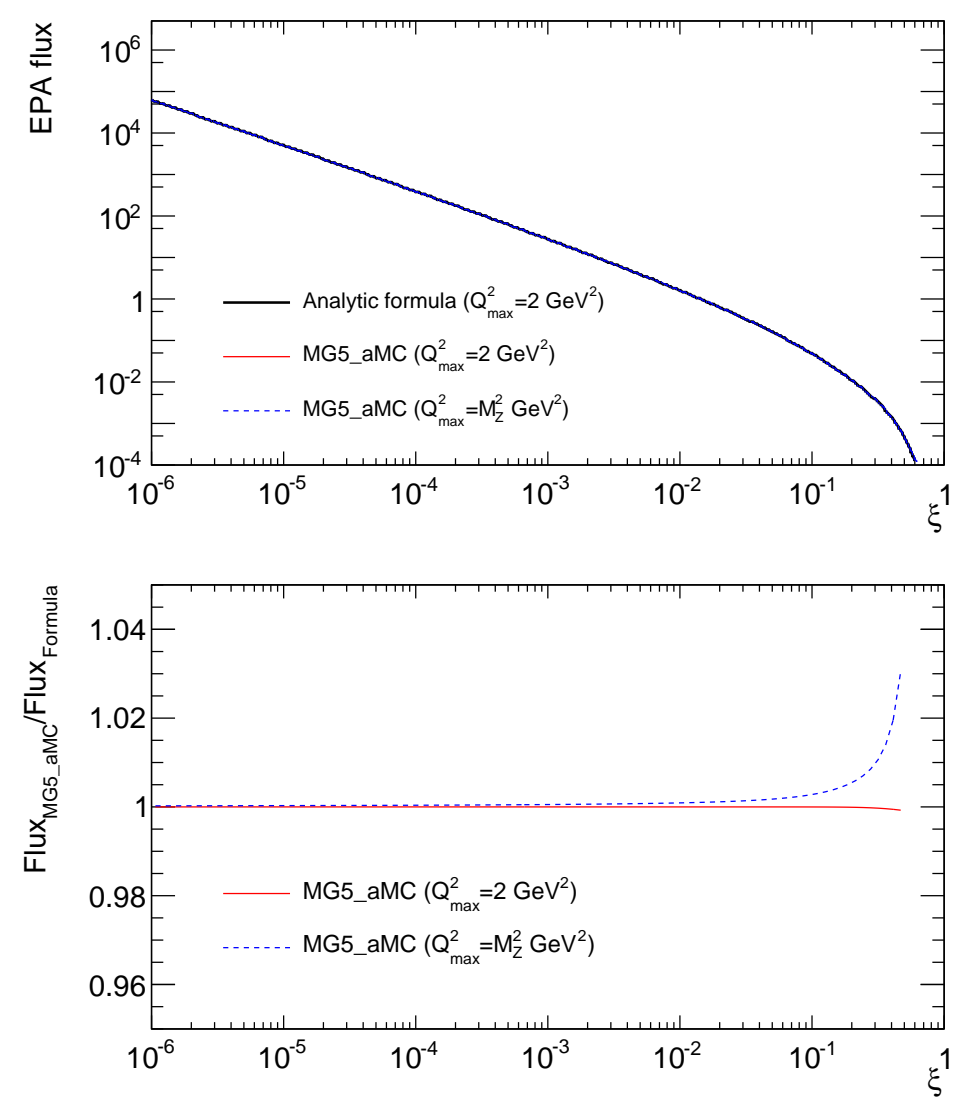

Figure 11: The EPA flux from a proton as a function of $\xi$, where the analytic expression in Eq. (17) with $Q_{\max }^{2}=2 \mathrm{GeV}^{2}$ (black) is compared with the numerical outcomes from MADGRAPH5_AMC@NLO for $Q_{\max }^{2}=2 \mathrm{GeV}^{2}$ (red) and $M_{Z}^{2} \mathrm{GeV}^{2}$ (blue). The ratios of the MADGRAPH5_AMC@NLO prediction to the analytic result are also shown in the bottom panel.

where $\xi=E_{\gamma} / E_{p}$ and $Q_{\max }^{2}$ are the energy fraction of the photon and the maximal value of the integration over the virtuality of the photon $Q^{2}$, respectively. Here, $Q_{\min }^{2}=m_{p}^{2} \xi^{2} /(1-\xi)$ and $Q_{0}^{2}=0.71 \mathrm{GeV}^{2}$. We usually choose $Q_{\max }^{2} \sim 2 \mathrm{GeV}^{2}$ since the contribution above a few $\mathrm{GeV}^{2}$ is negligible. The function $\varphi(x, y)$ is defined by

$$
\begin{aligned}
\varphi(x, y)= & (1+a z)\left[-\ln \left(\frac{1+y}{y}\right)+\sum_{k=1}^{3} \frac{1}{k(1+y)^{k}}\right]+\frac{(1-b) z}{4 y(1+y)^{3}} \\
& +\frac{c(4+z)}{4}\left[\ln \left(\frac{1+y-b}{1+y}\right)+\sum_{k=1}^{3} \frac{b^{k}}{k(1+y)^{k}}\right]
\end{aligned}
$$

with $z=x^{2} /(1-x)$. The parameters $a, b, c$ are related to the mass and the magnetic moment of the proton and the values are given as $a=7.16, b=-3.96$ and $c=0.028$.

The above structure function is implemented in MADGRAPH5_AMC@NLO [37 in a similar manner with PDF. In Fig. 11, we show the photon flux as a function of $\xi$, comparing the above analytic function with the numerical outcome of MADGRAPH5_AMC@NLO. 


\section{References}

[1] G. Aad et al. [ATLAS Collaboration], Phys. Lett. B 716, 1 (2012).

[2] S. Chatrchyan et al. [CMS Collaboration], Phys. Lett. B 716, 30 (2012).

[3] N. Arkani-Hamed, S. Dimopoulos and G. R. Dvali, Phys. Lett. B 429, 263 (1998).

[4] I. Antoniadis, N. Arkani-Hamed, S. Dimopoulos and G. R. Dvali, Phys. Lett. B 436, 257 (1998).

[5] L. Randall and R. Sundrum, Phys. Rev. Lett. 83, 3370 (1999).

[6] V. Khachatryan et al. [CMS Collaboration], arXiv:1408.3583 [hep-ex].

[7] G. Aad et al. [ATLAS Collaboration], arXiv:1502.01518 [hep-ex].

[8] V. Khachatryan et al. [CMS Collaboration], arXiv:1410.8812 [hep-ex].

[9] G. Aad et al. [ATLAS Collaboration], Phys. Rev. D 91, no. 1, 012008 (2015).

[10] S. Chatrchyan et al. [CMS Collaboration], Phys. Rev. Lett. 108, 111801 (2012).

[11] G. Aad et al. [ATLAS Collaboration], New J. Phys. 15, 043007 (2013).

[12] S. Chatrchyan et al. [CMS Collaboration], Phys. Lett. B 711, 15 (2012).

[13] G. Aad et al. [ATLAS Collaboration], Eur. Phys. J. C 74, no. 12, 3134 (2014).

[14] V. Khachatryan et al. [CMS Collaboration], arXiv:1411.2646 [hep-ex].

[15] S. Chatrchyan et al. [CMS Collaboration], Phys. Lett. B 720, 63 (2013).

[16] G. Aad et al. [ATLAS Collaboration], Phys. Rev. D 90, 052005 (2014).

[17] S. Chatrchyan et al. [CMS Collaboration], Phys. Rev. D 87, no. 11, 114015 (2013).

[18] G. Aad et al. [ATLAS Collaboration], Phys. Lett. B 718, 860 (2013).

[19] V. Khachatryan et al. [CMS Collaboration], JHEP 1408, 173 (2014).

[20] M. G. Albrow et al. [FP420 R\&D Collaboration], JINST 4, T10001 (2009).

[21] I. Sahin, A. A. Billur, S. C. Inan, B. Sahin, M. Köksal, P. Tektas, E. Alici and R. Yildirim, Phys. Rev. D 88, 095016 (2013).

[22] S. Fichet, G. von Gersdorff, B. Lenzi, C. Royon and M. Saimpert, JHEP 1502, 165 (2015). 
[23] G. F. Giudice, R. Rattazzi and J. D. Wells, Nucl. Phys. B 544, 3 (1999).

[24] T. Han, J. D. Lykken and R. J. Zhang, Phys. Rev. D 59, 105006 (1999).

[25] K. Hagiwara, J. Kanzaki, Q. Li and K. Mawatari, Eur. Phys. J. C 56, 435 (2008).

[26] J. L. Hewett, Phys. Rev. Lett. 82, 4765 (1999).

[27] H. Davoudiasl, J. L. Hewett and T. G. Rizzo, Phys. Rev. Lett. 84, 2080 (2000).

[28] J. Alwall, C. Duhr, B. Fuks, O. Mattelaer, D. G. Ozturk and C. H. Shen, arXiv:1402.1178 [hep-ph].

[29] P. de Aquino, K. Hagiwara, Q. Li and F. Maltoni, JHEP 1106, 132 (2011).

[30] C. Degrande, C. Duhr, B. Fuks, D. Grellscheid, O. Mattelaer and T. Reiter, Comput. Phys. Commun. 183, 1201 (2012).

[31] P. de Aquino, W. Link, F. Maltoni, O. Mattelaer and T. Stelzer, Comput. Phys. Commun. 183, 2254 (2012).

[32] N. D. Christensen, P. de Aquino, N. Deutschmann, C. Duhr, B. Fuks, C. Garcia-Cely, O. Mattelaer and K. Mawatari et al., Eur. Phys. J. C 73, no. 10, 2580 (2013).

[33] A. Alloul, N. D. Christensen, C. Degrande, C. Duhr and B. Fuks, Comput. Phys. Commun. 185, 2250 (2014).

[34] J. Alwall, R. Frederix, S. Frixione, V. Hirschi, F. Maltoni, O. Mattelaer, H.-S. Shao and T. Stelzer et al., JHEP 1407, 079 (2014).

[35] A. D. Martin, W. J. Stirling, R. S. Thorne and G. Watt, Eur. Phys. J. C 63, 189 (2009).

[36] M. R. Whalley, D. Bourilkov and R. C. Group, hep-ph/0508110.

[37] T. Pierzchala and K. Piotrzkowski, Nucl. Phys. Proc. Suppl. 179-180, 257 (2008).

[38] T. Sjostrand, S. Mrenna and P. Z. Skands, Comput. Phys. Commun. 178, 852 (2008).

[39] M. Cacciari, G. P. Salam and G. Soyez, JHEP 0804, 063 (2008).

[40] S. Fichet, G. von Gersdorff, O. Kepka, B. Lenzi, C. Royon and M. Saimpert, Phys. Rev. D 89 (2014) 11, 114004.

[41] V. M. Budnev, I. F. Ginzburg, G. V. Meledin and V. G. Serbo, Phys. Rept. 15, 181 (1975). 